REVISTAS DE LA FAHCE

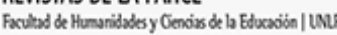

a 2
Educación Física y Ciencia

ISSN: 1514-0105

ISSN: 2314-2561

revistaefyc@fahce.unlp.edu.ar

Universidad Nacional de La Plata

Argentina

\title{
Determinação do tempo de inatividade pós-aquecimento de jogadores reservas em jogos de basquetebol
}

Santos, Sergio Costa; dos Santos Pinto, Herdley Kelvin; Bezerra de Almeida, Marcos

Determinação do tempo de inatividade pós-aquecimento de jogadores reservas em jogos de basquetebol

Educación Física y Ciencia, vol. 22, núm. 1, 2020

Universidad Nacional de La Plata, Argentina

Disponible en: http://www.redalyc.org/articulo.oa?id=439963095003

DOI: https://doi.org/10.24215/23142561e111

Esta obra está bajo una Licencia Creative Commons Atribución-NoComercial-Compartirlgual 4.0 Internacional. 


\section{Determinação do tempo de inatividade pós-aquecimento de jogadores reservas em jogos de basquetebol}

Determination of post-warm up inactivity time of non-starter players in basketball games

Sergio Costa Santos

Programa de Pós-Graduação em Educação Física da

Universidade Federal de Sergipe - Brasil (PPGEF/UFS).

Laboratório de Estudo e Pesquisa em Performance no

Exercicio e no Esporte, Brasil

ss_costa@outlook.com

Herdley Kelvin dos Santos Pinto

Laboratório de Estudo e Pesquisa em Performance no

Exercício e no Esporte - Brasil (L'Esporte). Departamento

de Educação Física da Universidade Federal de Sergipe,

Brasil

Marcos Bezerra de Almeida

Programa de Pós-Graduação em Educação Física da

Universidade Federal de Sergipe - Brasil (PPGEF/UFS).

Laboratório de Estudo e Pesquisa em Performance no

Exercício e no Esporte - Brasil (L'Esporte). Departamento

de Educação Física da Universidade Federal de Sergipe,

Brasil
DOI: https://doi.org/10.24215/23142561e111

Redalyc: http://www.redalyc.org/articulo.oa? id $=439963095003$

Recepción: 18 Junio 2019

Aprobación: 20 Enero 2020

\section{Resumo:}

Introdução: No basquetebol, atletas substitutos que entrem na partida apenas no segundo quarto de jogo (Q2) permanecem inativos por um longo, porém não objetivamente conhecido período de tempo. Objetivo: determinar a duração média do primeiro período (Q1) do basquetebol profissional, a duração média entre o término do aquecimento e início da partida (AQ-IP) e do segundo quarto (AQ-Q1), considerando diferentes competições e fases. Métodos: A amostra foi composta por 126 jogos de quatro competições Novo Basquete Brasil (NBB 2011/2012), American Cup2017, Eurobasket 2017 e Afrobasket 2017. Foram calculados os percentis de cada variável, além da comparação entre competições e entre fases da competição (fase classificatória e

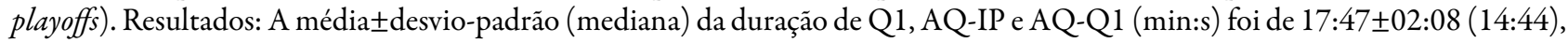
02:05 $\pm 00: 36$ (02:08) e 20:16 $\pm 02: 08$ (20:23), respectivamente. O Q1 dos jogos da Afrobasket foi mais longo que os do NBB $(.<0,05)$, mas não houve diferença entre a fase regular e os playoffs. Conclusão: Um atleta substituto que entre na partida apenas no Q2 permanece sentado no banco de reservas por mais de 20 minutos, independentemente da fase da competição. O Q1 dos jogos de seleções africanas é mais paralisado que os do campeonato brasileiro.

PalaVras-chaVE: Performance Esportiva, Exercício de Aquecimento, Regulamento, Atleta.

\section{Abstract:}

In basketball, substitute athletes who enter the match only in the second quarter of the game (Q2) remain inactive for a long, but not objectively known period of time. The objective of this paper is to determine a professional basketball first quarter (Q1) elapsed time, the time-interval from the end of warming-up period to the start of the game (AQ-IP), and to the start of the second quarter (AQ-Q1), considering different tournament and competition phases. The sample was composed by 126 matches of four tournaments: Novo BasqueteBrasil (NBB 2011/2012), American Cup 2017, Eurobasket 2017 e Afrobasket 2017. Statistical analysis determined percentiles for each variable, comparing among tournaments and between competition phases (regular season vs. playoff). The results shows that Mean \pm standard-deviation(median) of Q1, AQ-IP and AQ-Q1 (min:s) were 17:47 $\pm 02: 08$ 
(14:44), 02:05 $\pm 00: 36$ (02:08) e 20:16 $\pm 02: 08$ (20:23), respectively. Afrobasket games' Q1 were longer than NBB's ( $p<0.05)$, although no difference between regular season and playoffs were found. We can conclude that a non-starter basketball player that checks in only at Q2 remains seated on bench longer than 20 min, regardless of the competition phase. National African teams' Q1 matches are more interrupted than Brazilian teams'

KEYWORDs: Athletic Performance, Warm-Up Exercise, Regulation, Athlete.

\section{INTRODUÇÃO}

A relação benéfica entre aquecimento e desempenho atlético é investigada desde dos anos 1930 (Fradkin, Zazryn \& Smoliga, 2010). Informações mais detalhadas sobre o papel do exercício físico, sua relação com o aumento da temperatura corporal e o processo de dissipação de calor passam a ser reportadas a partir da década de 1960 (Saltin \& Hermansen, 1966). Expressivas evidências apresentadas até os dias atuais denotam a importância desse tema, assim como o consolidam como elemento indispensável a qualquer prática desportiva (Neiva et al., 2017; Racinais, Cocking, \& Périard, 2017).

Os benefícios imputados ao aquecimento abrangem aspectos psicológicos, prevenção de lesões e a melhoria da performance esportiva (Bishop, 2003; McCrary, Ackermann \& Halaki, 2015; McGowan, Pyne, Thompson \& Rattray, 2015; Sahin et al., 2018). Enquanto os benefícios de ordem psicológica parecem estar relacionados a um estado de prontidão e autoconfiança (Bishop, 2003; McGowan et al., 2015), os benefícios concernentes à prevenção de lesões são atrelados à adequação das amplitudes do movimento (Woods, Bishop \& Jones, 2007). Por fim, em que pese o desempenho, o aquecimento corporal tende a melhorar a coordenação motora, aumentar a oferta de oxigênio aos músculos, e melhorar a condução nervosa, entre outros (Bishop, 2003; Galazoulas, Tzimou, Karamousalidis \& Mougios, 2012).

Dessa forma, nota-se a importância dessas alterações quando consideradas as características do desporto moderno. As demandas fisiológicas, psíquicas e técnico-táticas são mais pronunciadas em atletas de alto rendimento (McLaren, Weston, Smith, Cramb \& Portas, 2016; Silva \& Tricoli, 2017). Isso faz com que o aquecimento seja um elemento imprescindível na preparação atlética atual (Fradkin et al., 2010; Racinais et al., 2017). Todavia, a preservação das alterações decorrentes do aquecimento depende da manutenção da temperatura corporal em níveis ótimos (McArdle, Katch \& Katch, 2011; Powers \& Howley, 2014), o que representa um desafio para atletas e técnicos quando intervalos de tempo entre o fim do período de aquecimento (AQ) e o início da competição propriamente dita é demasiadamente longo. Nesse sentido, robustas evidências apontam que um intervalo de 15 a 20 minutos é suficiente para que se sejam observadas quedas de performance (Alberti, Annoni, Ongaro, Scurati \& Michielon, 2014), sendo que a magnitude dessas perdas pode ser proporcional ao nível de desaquecimento (Galazoulas et al, 2012; West et al., 2013).

Essa é uma situação tipicamente encontrada em algumas modalidades esportivas, como decorrência de fatores ambientais e logísticos, tais como em provas de corridas de rua e regatas (Faulkner et al., 2012; Spitz, Kenefick \& Mitchell, 2014), ou mesmo por imposição das regras, como no caso do basquetebol (Federação Internacional de Basquete [FIBA], 2018). O artigo 9.5 da regra 4 do basquetebol determina que os jogadores só podem realizar aquecimento nos momentos que antecedem o primeiro (Q1) e terceiro (Q3) quartos do jogo. Portanto, os atletas substitutos devem permanecer sentados no banco de reservas durante todo o jogo enquanto não forem solicitados a entrar em quadra.Em decorrência disso, um atleta substituto que entre na partida apenas no segundo quarto $(\mathrm{Q} 2)$ do jogo permanece sentado em repouso ao longo do período compreendido entre o AQ e o início do Q2.

Contudo, é importante frisar que a duração desse período não é objetivamente conhecida, sendo limitada a especulações e estimativas. Por exemplo, Galazoulas et al. (2012) consideraram a duração do primeiro tempo (Q1 + Q2) arbitrariamente igual a 40 minutos, embora não tenham apresentado critérios para sustentar essa definição. Assim, com base em uma perspectiva ecológica, faz-se necessário conhecer o tempo de inatividade a que um atleta pode ser submetido antes de sua efetiva participação no jogo, visto que esse período pode 
impactar outros aspectos relacionados ao jogo, como manutenção de temperatura corporal, desempenho esportivo ou mesmo risco de lesões, entre outros.

Dessa forma, o presente estudo tem a finalidade de conhecera duração efetiva de três intervalos de tempo em jogos oficiais de basquetebol: a) entre o fim do aquecimento e o início da partida (AQ-IP); b) a duração total do primeiro quarto (Q1); e c) entre o fim do aquecimento e o fim do primeiro quarto (AQ-Q1), considerando diferentes competições e fases (classificatória e playoff).

\section{Metodologia}

Este estudo é caracterizado como uma pesquisa descritiva de análise observacional, e foram contemplados jogos de basquetebol profissional tanto em nível nacional (Novo Basquete Brasil - NBB temporada 2011/2012), como internacional (Copa América de Basquetebol - American Cup 2017, Copa Europeia de Basquetebol - Eurobasket 2017, e Copa Africana de Basquetebol - Afrobasket 2017), todas do naipe masculino. Os jogos do NBB estavam armazenados em DVD, tendo sido gravados ao vivo durante as transmissões pela TV, e faziam parte do acervo pessoal de um dos pesquisadores. Os jogos das Copas Continentais (América, África e Europa) foram também gravados em DVD para durante a realização das respectivas competições entre os meses de agosto e setembro de 2017. Foi registrado exclusivamente o intervalo de tempo entre o fim do AQ e o início da partida,e o tempo total do Q1 de um jogo de basquetebol. O projeto foi aprovado pelo Comitê de Ética em Pesquisa institucional local (parecer $\mathrm{n}^{\circ} 2.802 .375$ ).

A amostra foi composta por 126 jogos, sendo eles distribuídos como: 49 jogos do NBB 2011/2012, 50 jogos da Eurobasket 2017, 12 jogos da American Cup e 15 jogos da Afrobasket 2017.Os jogos do NBB foram escolhidos por representar a elite do basquetebol brasileiro. As competições continentais representam equipes de três escolas (estilos de jogo) reconhecidamente distintas, o que poderia resultar em diferenças nos aspectos temporais do jogo. $\mathrm{O}$ número de jogos varia em cada competição em decorrência do número de equipes participantes, sendo que todas apresentaram fase classificatória e fase de playoff. Como critérios de seleção, foram considerados os jogos transmitidos ao vivo por um canal de TV por assinatura ou por uma plataforma digital da internet. Como critérios de exclusão, foram desconsiderados os jogos em que tenha acontecido algum problema técnico que retardasse o andamento natural da partida ou que impedisse sua gravação (cinco jogos descartados).

Os jogos foram gravados em um dispositivo de armazenamento para posterior análise, e a decupagem das gravações foi feita utilizando-se uma planilha para registro do tempo decorrido entre o fim do AQ e o início da partida e durante o Q1. A duração do tempo corrido de jogo, os momentos de início e fim do Q1 foram determinados, respectivamente, da seguinte forma: a) quando a bola foi tocada pela primeira vez após o árbitro lançá-la ao alto para o início da partida; e b) quando o cronômetro de jogo foi zerado, simultaneamente ao sinal sonoro da mesa de arbitragem. Os jogos gravados contemplaram as fases classificatórias e playoffs de cada competição.

Para análise do intervalo de tempo entre o fim do AQ e o início do jogo (AQ-IP), foram considerados dois momentos: a) fim do período de aquecimento (determinado pelo árbitro da partida); b) o início do Q1. O tempo total entre o AQ e o final de Q1 (AQ-Q1) foi determinado pela soma dos valores do AQ e do tempo corrido do Q1. A medida do tempo foi feitacom cronômetro digital e os dados foram registrados em planilha eletrônica.

Os dados foram analisados de forma descritiva por média \pm desvio padrão (valor mínimo a valor máximo), e a determinação dos percentis 90 (P90), 75 (P75), 50 (P50), 25 (P25) e 10 (P10). O teste de KolmogorovSmirnov indicou que a distribuição dos dados do intervalo AQ-IP não era normal $(p=0,006), \operatorname{logo} a$ comparação entre os campeonatos foi feita pelo teste não-paramétrico de Kruskal-Wallis, e a comparação entre as fases classificatória e playoff foi feita pelo teste de Mann-Whitney. As durações de Q1 e do intervalo AQ-Q1 apresentaram distribuição normal ( $\mathrm{p}=0,200$ para ambos), e, portanto, as comparações foram feitas 
pela ANOVA de uma entrada com post hoc de Bonferroni, e as fases da competição pelo teste $t$ de Student. O teste de Levene denotou homogeneidade das variâncias apenas da duração do $Q 1(p=0,725)$ e do intervalo AQ-Q1 ( $p=0,669)$. Em todos os casos, foi aceito um nível de significância de 5\%. O software estatístico SPSS 20.0 (IBM, EUA) foi utilizado para todos os cálculos.

\section{Resultados}

Por limitações operacionais, não foi computado o momento exato em que o aquecimento era finalizado nos jogos do NBB. Desta forma, os intervalos AQ-IP e AQ-Q1 deste campeonato não foram considerados

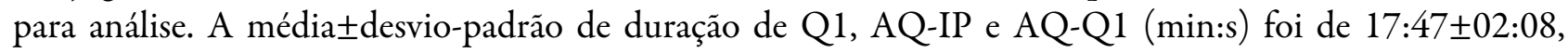

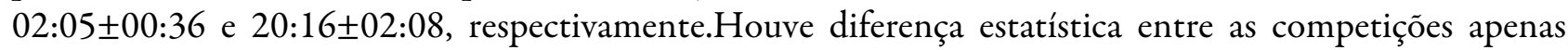
quanto à duração do Q1, sendo mais longa nos jogos da Afrobasket em comparação com os do NBB (tabela 1).

Tabela 1. Comparação das médias \pm desvios-padrões das durações do intervalo AQ-IP, do Q1 e do intervalo AQ-Q1 entre as diferentes competições. Dados são apresentados em minutos: segundos.

\begin{tabular}{lccccc}
\hline & NBB $^{n}$ & Eurobasket & Afrobasket & Copa América & $p$ \\
\hline AQ-IP & -- & $02: 01 \pm 00: 32$ & $02: 12 \pm 00: 49$ & $02: 19 \pm 00: 38$ & 0,493 \\
Q1 & $17: 12 \pm 02: 00$ & $18: 10 \pm 02: 10$ & $18: 46 \pm 02: 27^{*}$ & $17: 37 \pm 01: 48$ & 0,046 \\
AQ-Q1 & -- & $20: 11 \pm 02: 12$ & $20: 51 \pm 02: 16$ & $19: 56 \pm 01: 39$ & 0,478 \\
\hline
\end{tabular}

aNão foi possível identificar o momento em que o aquecimento era

finalizado nos jogos do NBB. ${ }^{*}$ significa $\mathrm{p}<0,05$ em relação ao NBB.

A distribuição percentil dos tempos de jogo investigados mostrou que a duração do Q1 ultrapassa os 17 minutos, o que faz com queo início do Q2 ocorradepois de ultrapassadosmais de20 minutosdo término do aquecimento em pelo menos metade dos casos (tabela 2).

Tabela 2. Distribuição dos percentis das durações do intervalo AQ-IP, do

Q1 e do intervalo AQ-Q1. Dados são apresentados em minutos: segundos.

\begin{tabular}{lccccc}
\hline & P90 & P75 & P50 & P25 & P10 \\
\hline AQ-IP & $02: 46$ & $02: 25$ & $02: 08$ & $01: 49$ & $01: 00$ \\
Q1 & $20: 24$ & $19: 19$ & $17: 44$ & $16: 14$ & $15: 05$ \\
AQ-Q1 & $22: 49$ & $21: 38$ & $20: 23$ & $18: 49$ & $17: 39$ \\
\hline
\end{tabular}

Não houve diferença na duração dos intervalos de tempo entre os jogos disputados nas fases classificatória e de playoff (tabela 3). 
Tabela 3. Comparação das médias \pm desvios-padrões das durações do intervalo AQ-IP, do Q1 e do intervalo AQ-Q1 entre as fases de competição. Dados são apresentados em minutos: segundos.

\begin{tabular}{lccc}
\hline & Classificatória & Playoff & $p$ \\
\hline AQ-IP & $02: 05 \pm 00: 39$ & $02: 07 \pm 00: 32$ & 0,897 \\
Q1 & $17: 44 \pm 02: 16$ & $17: 56 \pm 01: 57$ & 0,632 \\
AQ-Q1 & $20: 16 \pm 02: 16$ & $20: 17 \pm 01: 49$ & 0,618 \\
\hline
\end{tabular}

\section{Discussão}

O objetivo do presente estudo foiinvestigar o tempo corrido de um primeiro quarto (Q1) de uma partida de basquetebol, o intervalo entre o fim do aquecimento e o início do primeiro quarto da partida (AQ-IP) em diferentes competições e fases. Emborano papel a duração de um quarto seja de 10 min (FIBA, 2018), as condicionantes impostas pelas regras determinam que o cronômetro deve ser parado automaticamente a cada falta, violação e tempo técnico.Dessa forma, a duração média do Q1 oscilou entre 17 e 18 min, sendo mais longo na Afrobasket (margeando os $19 \mathrm{~min}$ ), e mais curto nos jogos do NBB (pouco acima de $17 \mathrm{~min}$ ), independentemente da fase da competição.

É importante destacar que, apesar de não estar oficialmente escrito em normas, os árbitros de basquetebolsão orientados a controlar o tempode intervalo entre o fim doAQe o início Q1. Pela determinação, as equipes têm um minuto para se organizar, ou seja, estar em quadra com apenas os cinco jogadores titulares, e pronta para iniciar a partida.Porém, essa orientação parece ser raramente seguida,sendo observadosperíodos de até 4 minutos desse intervalo (dados não apresentados). A partir da análise de todas as partidas disponíveis, a duração de AQ-IP foi em torno de 2 minem pelo menos metade das partidas (percentil 50), o que equivale ao dobro do tempo determinado pela FIBA.

Esses resultados representam a condição que mais se apresenta no contexto de uma partida oficial de basquetebol, visto que foram englobadas todas as paralisações do cronômetro por intervenção da equipe de arbitragem. Entre a primeira e a segundametades do jogo identifica-se uma redução da prevalência de intervalos de tempo com bola viva (cronômetro livre) e um simultâneo aumento da prevalência de intervalos de bola morta (cronômetro pausado) (Conte, Tessitore, Smiley, Thomas \& Favero, 2016). Contudo, ao analisar os dados de Borin, Gonçalves, Padovani \& Aragon (2005), observa-se que o número de paralisações não muda de forma expressiva ao longo dos quartos de partida, variando entre 13 e 17 intervenções dos árbitros. Quanto maior a quantidade de paralisações, associado ao tempo de permanência em inatividade, maior será o tempo total decorrido na partida. Assim, pode-se atestar o real tempo em que um jogador substituto, em respeitoaoartigo 9.5 da regra 4 (FIBA, 2018), passaria sentado no banco de reservas de sua equipe após o término do AQ.

A precisão desse tempo de inatividade faz-se importante para se questionar se o estado de prontidão para a atividade competitiva,obtido viaaquecimento,ainda estaria (Boudenot, 2018). A presença dessas alterações pode ser determinante para a performance e/ou permanência desse atleta na partida (Russell et al., 2015; McGowan, Thompson, Pyne, Raglin \& Rattray, 2015), tendo em vista que a literatura tem apresentado resultados robustos da ação benéfica do aquecimento prévio sobre a performance atlética, estado psicológico e prevenção de lesões (Woods et al., 2007; Almeida et al., 2009; McGowan et al., 2015a). Entretanto, a ampla maioria dessas alterações é dependente do aquecimento e/ou sua manutenção, ou seja, em virtude do 
desaquecimento esses benefícios seriam gradativamente dissipados (McGowan et al., 2015b; Russell et al., 2015).

A relação conflitante entre os efeitos do aquecimento versus desaquecimento pode ser verificada no estudo de Neiva et al. (2017), que investigou o desempenho de nadadores após intervalos de 10 e 20 min. Nesse estudo, os atletas apresentaram performances melhores $10 \mathrm{~min}$ pós-aquecimento do que após 20 min. Corroborando com esses resultados, Galazoulas et al. (2012) submeteram atletas de basquetebol masculino efeminino a intervalos de 10, 20,30 e 40 min após sessão específica de aquecimento. Os resultados apresentaram declíniosem testes físicos (potência muscular e velocidade de deslocamento) proporcionais ao tempo de inatividade, apresentando maiores perdas na performance após $40 \mathrm{~min}$ de intervalo.

Esse conjunto de resultados aponta tendência a prejuízosno desempenho já a partir de $10 \mathrm{~min}$ de inatividade. Tal assertiva é fortalecida por Russel et al. (2015) que identificaram perdas de rendimento em atletas de rugby após o intervalo entre o primeiro e segundo tempos da partida. Os autores também atribuíram essas perdas ao intervalo de descanso de 15 minentre as etapas da partida como suficiente para que o desempenho tanto físico como cognitivo fosse comprometido. Dessa forma, ressaltam a necessidade de se estabelecer estratégias de manutenção ou retomada do aquecimento durante o intervalo.

Assim, os cerca de 18 min encontrados como duração média do Q1 representam uma condição adversa ao atleta que permanece sentado no banco de reservas por todo esse quarto de partida. Deve-se levar em conta que no decorrer da partida os jogadoresrealizam um amplo conjunto de movimentos, tais como corridas de velocidades e distâncias variadas, com acelerações, desacelerações e paradas bruscas, mudanças de direção e saltos, além de ações técnicas específicas (McInnes, Carlson, Jones \& McKenna, 1995; Ben Abdelkrim et al., 2010). Além disso, é importante frisar que o jogador deve manter altos níveis de atividade enquanto estiver em quadra, tanto na defesa como no ataque (Ziv \& Lidor, 2009). A rápida transição de um estado de inércia (banco de reservas) para realização de movimentos rápidos em alta intensidade (Conte et al., 2015) pode causar lesões (Castro, Marques, Hallal \& Gonçalves, 2017). Portanto, nessa condição, essas constantes demandas físicas, psíquicas e cognitivas podem representar mais um desafio à performance do atleta (Silva \& Tricoli, 2017; McGowan et al., 2015a).

Face a esses desafios, vê-se que o sucesso e a permanência desses atletas na partida dependerão de um estado ótimo de desempenho que é conseguido através dos efeitos preventivos e ergogênicos desencadeados e/ou mantidos por práticas prévias de aquecimento (Neiva et al., 2017; Fradkin et al., 2010; McCrary et al., 2015). Por conseguinte, essa condição também potencializaria as chances de vitória do atleta e sua equipe frente aos adversários (McGowan et al., 2015a). Assim, pode-se observar a estreita relação entre o aquecimento, a preparação atlética e o desempenho.

Apesar de a literatura existente apresentar resultados consistentes sobre a relação aquecimento e desempenho atlético, não foram encontrados estudos que abordassem especificamente a condicionante imposta pelo artigo 9.5 da regra 4 do basquetebol (FIBA, 2018). Assim, o presente estudo pode servir como base para determinar o real tempo que um atleta substituto que entraria na partida ao iniciar o Q2 passaria em condição de inatividade. Esse resultado pode dar suporte à determinação de estratégias para atletas, técnicos e/ou envolvidos no processo de preparação atlética, no sentido de aproveitar todas as alteraçóes positivas desencadeadas pelo aquecimento prévio.

\section{Conclusão}

Com base nas competições analisadas (NBB, Eurobasket, Afrobasket, Copa América) conclui-se que o tempo que um atleta substituto permanece sentado no banco de reservas de sua equipe entre o AQ e o fim do Q1 é de aproximadamente 20 minutos. Importante ressaltar que esse cenáriose aplica ao jogador substituto que entre na partida ao iniciar o Q2, ou seja, pode-se assumir que esse tempo de inatividade seja ainda mais prolongado para aqueles substitutos que entrem nos jogos apenas no decorrer do Q2. 


\section{REFERENCIAS}

Alberti, G., Annoni, M., Ongaro, L., Scurati, R. \& Michielon, G. (2014). Athletic performance decreases in young basketball players after sitting. International Journal of Sports Science \& Coaching, 9(5), 975-84.

Almeida, PHF, Barandalize, D, Ribas, DIR, Gallon, D, Macedo, ACB \& Gomes, ARS. (2009). Alongamento Muscular: suas implicações na performance e na prevenção de lesões. Fisioterapiaem Movimento, 22(3), 335-43.

Ben Abdelkrim, N, Castagna, C,Jabri, I, Battikh, T, El Fazaa, S \& El Ati, J. (2010). Activity profile and physiological requirements of junior elite basketball players in relation to aerobic-anaerobic fitness. Journal of Strength and Conditioning Research, 24(9), 2330-42.

Bishop, D. (2003). Warm Up I: Potential mechanisms and the effects of passive warm up on exercise performance. Sports Medicine, 33(6), 439-54.

Borin, JP, Gonçalves, A, Padovani, CR \& Aragon, FF. (2005). Perfil da intensidade de esforço nas ações e nos tempos do jogo de basquete de alto nível. Salusvita, 24(3), 411-8.

Boudenot, A. (2018). Training and competition warm up approaches. Science \& Sports, 33(3), 137-44.

Castro, A., Marques, NR, Hallal, CZ \& Gonçalves, M. (2017). Órtese de tornozelo não influencia a força e o equilíbrio funcional dos músculos do tornozelo durante exercício em intensidade do jogo de basquetebol. Revista Brasileira de Educação Física e Esporte, 31(1), 71-81.

Conte, D, Favero, TG, Lupo, C, Francioni, FM, Capranica, L \& Tessitore, A. (2015). Time-motion analysis of Italian elite women's basketball games: individual and team analyses. Journal of Strength and Conditioning Research, 29(1), 144-50.

Conte, D, Tessitore, A, Smiley, K, Thomas, C \& Favero, TG. (2016). Performance profile of NCAA Division I men's basketball games and training sessions. Biology of Sport, 33(2), 189-94.

Faulkner, SH, Ferguson, RA, Gerrett, N, Hupperets, M, Hodder, SG \& Havenith, G. (2012). Reducing muscle temperature drop post warm-up improves sprint cycling performance. Medicine and Science in Sports and Exercise, 45(2), 359-65.

Federação Internacional de Basquete (FIBA). (2018). Official Basketball Rules - 2018. Mies, Switzerland: FIBA Central Board. Recuperado de: http://www.fiba.basketball/documents/official-basketball-rules.pdf.

Fradkin, AJ, Zazryn, TR \& Smoliga, JM. (2010). Effects of warming-up on physical performance: a systematic review with meta-analysis. Journal of Strength and Conditioning Research, 24(1), 140-8.

Galazoulas, C, Tzimou, A,Karamousalidis, G \& Mougios, V. (2012). Gradual decline in performance and changes in biochemical parameters of basketball players while resting after warm-up. European Journal of Applied Physiology, 112(9), 3327-34.

McArdle, WD, Katch, FI \& Katch, VL. (2011). Fisiologia do exercício: nutrição, energia e desempenho humano (7ed.). Rio de Janeiro: Guanabara Koogan.

McCrary, JM, Ackermann, BJ \& Halaki, M. (2015). A systematic review of the effects of upper body warm-up on performance and injury. British Journal of Sports Medicine, 49(14), 935-42.

McGowan, CJ, Pyne, DB, Thompson, KG \& Rattray, B. (2015). Warm-up strategies for sport and exercise: mechanisms and applications. Sports Medicine, 45(11), 1523-46.

McGowan, CJ, Thompson, KG, Pyne, DB, Raglin, JS \& Rattray, B. (2015). Heated jackets and dryland-based activation exercises used as additional warm-ups during transition enhance sprint swimming performance. Journal of Science and Medicinein Sport, 19(4), 354-8.

McInnes, SE, Carlson, JS, Jones, CJ \& McKenna, MJ. (1995). The physiological load imposed on basketball players during competition. Journal of Sports Science, 13(5), 387-97.

McLaren, SJ, Weston, M, Smith, A, Cramb, R \& Portas, MD. (2016). Variability of physical performance and player match loads in professional rugby union. Journal of Science and Medicine in Sport, 19(6), 493-97. 
Neiva, HP, Marques, MC, Barbosa, TM, Izquierdo, M , Viana, JL \& Marinho, DA . (2017). Effects of 10 min vs. 20 min passive rest after warm-up on $100 \mathrm{~m}$ freestyle time-trial performance: A randomized crossover study.Journal of Science and Medicine in Sport, 20(1), 81-6.

Powers, SK \& Howley, ET. (2014). Fisiologia do exercício: teoria e aplicação ao condicionamento e ao desempenho (8ed.). Barueri: Manole.

Racinais, S, Cocking, S \& Périard, JD. (2017). Sports and environmental temperature: from warming-up to heatingup. Temperature (Austin), 4(3), 227-57.

Russell, M, West, DJ, Briggs, MA, Bracken, RM, Cook, CJ, Giroud, T,...Kilduff, LP. (2015). A passive heat maintenance strategy implemented during a simulated half-time improves lower body power output and repeated sprint ability in professional rugby union players. PloS One, 10(3), e0119374.

Sahin, N, Gurses, VV, Baydil, B, Akgul, MS, Feka, K, Iovane, A \& Messina, G. (2018). The effect of comprehensive warm up (FIFA 11+Program) on motor abilities in young basketball players: a pilot study. ActaMedicaMediterranea, 34(3), 703-7.

Saltin, B \& Hermansen, L. (1966). Esophageal, rectal, and muscle temperature during exercise. Journal of Applied Physiology, 21(6), 1757-62.

Silva, CMP \& Tricoli, V. (2017). Características fisiológicas do basquetebol. In D. De Rose Jr. \& V. Tricoli (Eds.), Basquetebol do treino ao jogo (2ed., pp. 15-26).Barueri: Manole.

Spitz, MG, Kenefick, RW \& Mitchell, JB. (2014). The effects of elapsed time after warm-up on subsequent exercise performance in a cold environment. Journal of Strength and Conditioning Research, 28(5), 1351-57.

West, DJ, Dietzig, BM, Bracken, RM, Cunningham, DJ, Crewther, BT, Cook, CJ \& Kilduff, LP. (2013). Influence of post-warm-up recovery time on swim performance in international swimmers. Journal of Scienceand Medicine in Sport, 16(2), 172-76.

Woods, K, Bishop, P \& Jones, E. (2007). Warm-up and stretching in the prevention of muscular injury. Sports Medicine, 37(12), 1089-99.

Ziv, G \& Lidor, R. (2009). Physical attributes physiological characteristics, on-court performances and nutritional strategies of female and male basketball players. Sports Medicine, 39(7), 547-68.

\section{BY-NC-SA}

